\title{
An Optically Tunable Optoelectronic Oscillator
}

\author{
Wangzhe Li, Student Member, IEEE, and Jianping Yao, Senior Member, IEEE, Fellow, OSA
}

\begin{abstract}
An optically tunable optoelectronic oscillator (OEO) implemented by employing a two-port optical phase modulator without using any electronic microwave filters is proposed and experimentally demonstrated. The key device in the system is the two-port phase modulator, which functions, in conjunction with a dispersive element in the loop, to form a high-Q microwave filter to perform microwave frequency selection. The central frequency of the microwave filter is a function of the optical wavelength and the chromatic dispersion of the dispersive element, therefore, the oscillation frequency can be simply tuned by tuning the wavelength of the laser source or the chromatic dispersion of the dispersive element. A theoretical analysis is provided, which is verified by experiments. The phase noise performance and the frequency tunability are both experimentally investigated.
\end{abstract}

Index Terms-Chromatic dispersion, microwave generation, microwave photonics, optoelectronic oscillator (OEO), phase modulation.

\section{INTRODUCTION}

A N optoelectronic oscillator (OEO) [1] with frequency tunability can find numerous applications such as in optical and wireless communications [2], [3], radar, and modern instrumentation [4]. In an OEO, the loop length is usually from a few meters to tens of kilometers, which makes the OEO to have densely spaced oscillation modes. To ensure an OEO to operate at a single oscillation mode, a high Q microwave filter is usually needed to perform the mode selection [5]. The use of multiple loops in an OEO would make the mode spacing greater, which would ease the requirement for a high $\mathrm{Q}$ microwave filter [6]-[8]. However, an OEO with multiple loops would have a poorer system stability and higher system cost. More importantly, the frequency tunability would be complicated and the tunable range is small if a multiple-loop structure is employed. For example, a continuously frequency tunable OEO was proposed in [9], in which a wavelength-tunable laser source and two dispersive optical fiber loops were employed. The frequency tuning was realized by tuning the wavelength of the laser source. Due to the wavelength-dependent loop dispersion or loop delay, the mode spacing was tuned, leading to the tuning of the oscillating frequency. Since the tunable range of the mode spacing was limited to a few $\mathrm{MHz}$, The frequency tunable range was only a few MHz.

Manuscript received January 26, 2010; revised May 31, 2010; accepted July 05, 2010. Date of publication July 15, 2010; date of current version August 23, 2010. This work was supported by the Natural Sciences and Engineering Research Council of Canada (NSERC).

The authors are with the Microwave Photonics Research Laboratory, School of Information Technology and Engineering, University of Ottawa, Ottawa, ON K1N 6N5, Canada (e-mail: jpyao@site.uottawa.ca).

Color versions of one or more of the figures in this paper are available online at http://ieeexplore.iee.org.

Digital Object Identifier 10.1109/JLT.2010.2058792

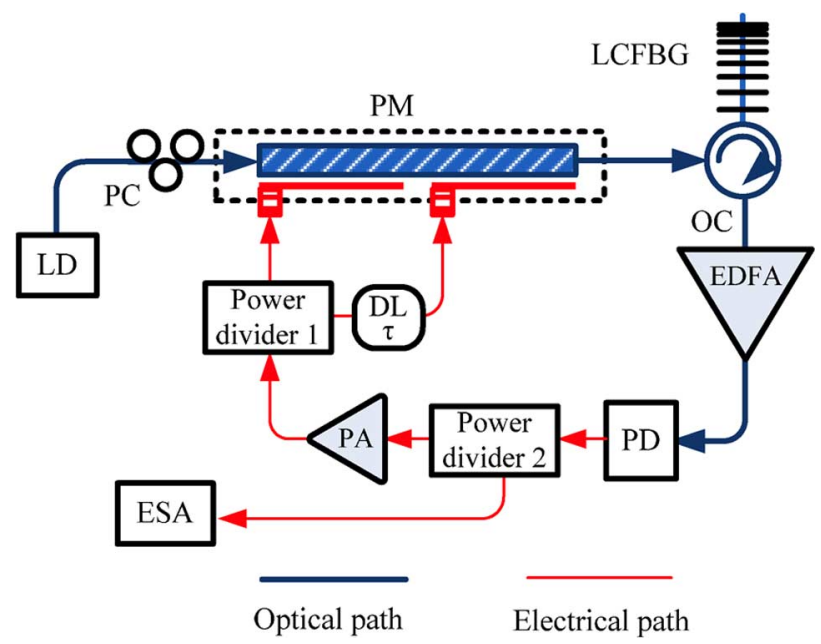

Fig. 1. Schematic diagram of the proposed frequency-tunable OEO.

In this paper, we propose and demonstrate an optically tunable OEO implemented by employing a two-port optical phase modulator (PM) without using any electronic microwave filters. The key device in the system is the two-port PM, which functions, in conjunction with a dispersive element in the loop, to form a high-Q microwave filter to perform microwave frequency selection [10], [11]. The dispersive device employed can be a linearly chirped fiber Bragg grating (LCFBG), a dispersion compensating fiber or even a single-mode fiber (SMF). The central frequency of the microwave filter is a function of the optical wavelength of the laser source and the chromatic dispersion of the dispersive element, therefore, the oscillation frequency can be simply tuned by tuning the wavelength of the laser source or the dispersion of the dispersive element. The key significance of the proposed approach is that no electronic microwave filters are employed, which ensures a simplified configuration and an increased frequency tunable range. In addition, the PM is not biased, which would eliminate the instability caused by the bias drifts which exists in an intensity-modulator-based system. A theoretical analysis is provided, which is verified by experiments. Single frequency oscillation is observed and frequency tunability is demonstrated. The phase noise of the generated signal is also investigated.

\section{PRINCIPLE OF OPERATION}

The configuration of the proposed single-loop frequency-tunable OEO is shown in Fig. 1. The system consists of a laser diode (LD), a polarization controller (PC), a two-port PM, a dispersive element which is an LCFBG in the configuration, an optical circulator (OC), an erbium-doped fiber amplifier (EDFA), a photodetector (PD), two RF power dividers, an RF power amplifier (PA), and an RF delay line (DL). The light wave from the LD is sent to the PM, which is modulated by the oscillating signal at 
the PM, and then sent to the LCFBG. The LCFBG here is functioning as a dispersive element, to convert the phase-modulated signal to an intensity-modulated signal. After optical amplification by the EDFA and electrical amplification by the PA, the oscillating signal is split into two paths and applied to the PM via the two RF ports. A time delay difference between the two ports is introduced by using a DL in one path.

There are three different delay times that support the single frequency operation of the OEO: the total feedback loop delay, the RF delay between the two ports of the PM, and the delay introduced by the dispersive element. The functions of the three delays are different. The first delay introduces a phase increment to each of the frequency components after each circulation. The RF delay between the two ports of the PM introduces a frequency-dependent loss. The dispersive element and the PM form an equivalent microwave bandpass filter. Only the frequency component with a phase increment after each circulation being a multiple of $2 \pi$ and with the smallest loss can oscillate.

It is known that a phase-modulated signal can be converted to an intensity-modulated signal at a dispersive fiber, and the phase modulation to intensity modulation (PM-IM) conversion has a transfer function corresponding to a microwave bandpass filter [10], [11]. Assume that a one-port PM is driven by a microwave signal $V_{\mathrm{e}} \cos \omega_{\mathrm{e}} t$, where $V_{e}$ is the amplitude and $\omega_{e}$ is the angular frequency of the input microwave signal, the electrical field $E_{1}(t)$ at the output of the PM can be expressed as

$$
\begin{aligned}
E_{1}(t) & =E_{0} \exp \left\{j\left[\omega_{\mathrm{o}} t+\frac{V_{\mathrm{e}}}{V_{\pi}} \cos \left(\omega_{\mathrm{e}} t\right)\right]\right\} \\
& =E_{0} \exp \left(j \omega_{\mathrm{o}} t\right) \sum_{n=-\infty}^{\infty} j^{n} \cdot J_{n}(\gamma) \cdot \exp \left(\mathrm{jn} \omega_{\mathrm{e}} \mathrm{t}\right)
\end{aligned}
$$

where $E_{0}$ is amplitude of the electrical field and $\omega_{o}$ is the angular frequency of the incident light wave, $V_{\pi}$ is the half wave voltage of the PM, $J_{n}$ is the $n$ th-order Bessel function of the first kind, $\gamma=V_{e} / V_{\pi}$ is the phase modulation index. Under small signal modulation condition, (1) can be rewritten as

$$
\begin{array}{r}
E_{1}(t) \approx E_{0}\left[J_{0}(\gamma) \cos \left(\omega_{\mathrm{o}} t\right)+J_{1}(\gamma) \cos \left(\omega_{\mathrm{o}} t+\omega_{\mathrm{e}} t+\pi / 2\right)\right. \\
\left.-J_{1}(\gamma) \cos \left(\omega_{\mathrm{o}} t-\omega_{\mathrm{e}} t-\pi / 2\right)\right] .
\end{array}
$$

From (2), we can see there are only three main spectral components: the optical carrier at $\omega_{\mathrm{o}}$, the upper sideband at $\omega_{0}+\omega_{e}$ and the lower sideband at $\omega_{o}-\omega_{e}$. After transmission over a fiber, the optical field would be

$$
\begin{aligned}
E_{2}(t)= & E_{0}\left[J_{0}(\gamma) \cos \left(\omega_{\mathrm{o}} t+\theta_{0}\right)\right. \\
& +J_{1}(\gamma) \cos \left(\omega_{\mathrm{o}} t+\omega_{\mathrm{e}} t+\pi / 2+\theta_{1}\right) \\
& \left.-J_{1}(\gamma) \cos \left(\omega_{\mathrm{o}} t-\omega_{\mathrm{e}} t-\pi / 2+\theta_{2}\right)\right]
\end{aligned}
$$

where $\theta_{i}(i=0,1,2)$ represents the additional phase introduced by the fiber. We have $\theta=\beta L$, where $\beta$ is the propagation constant, $L$ is the length of the fiber. When expanding $\beta$ in a Taylor series, we have

$$
\begin{aligned}
& \theta_{0}=L \beta\left(\omega_{\mathrm{o}}\right) \\
& \theta_{1}=L \beta\left(\omega_{\mathrm{o}}\right)+L \dot{\beta}\left(\omega_{\mathrm{o}}\right) \omega_{\mathrm{e}}+L \ddot{\beta}\left(\omega_{\mathrm{o}}\right) \omega_{\mathrm{e}}^{2} / 2
\end{aligned}
$$

$$
\begin{aligned}
& \approx L \beta\left(\omega_{\mathrm{o}}\right)+L n_{\mathrm{o}} \omega_{\mathrm{e}} / c-L D \lambda^{2} \omega_{\mathrm{e}}^{2} / 4 \pi c \\
\theta_{2} & =L \beta\left(\omega_{\mathrm{o}}\right)-L \dot{\beta}\left(\omega_{\mathrm{o}}\right) \omega_{\mathrm{e}}+L \ddot{\beta}\left(\omega_{\mathrm{o}}\right) \omega_{\mathrm{e}}^{2} / 2 \\
& \approx L \beta\left(\omega_{\mathrm{o}}\right)-L n_{\mathrm{o}} \omega_{\mathrm{e}} / c-L D \lambda^{2} \omega_{\mathrm{e}}^{2} / 4 \pi c
\end{aligned}
$$

where $\beta\left(\omega_{\mathrm{o}}\right), \dot{\beta}\left(\omega_{\mathrm{o}}\right)$ and $\ddot{\beta}\left(\omega_{\mathrm{o}}\right)$ are the zeroth-, first- and second-order derivatives of the propagation constant at $\omega_{o}, D$ is the chromatic dispersion parameter of the fiber, $c$ is the velocity of light in vacuum, $n_{o}$ is the refractive index of the fiber, and $\lambda$ is the wavelength of the incident light wave. Then, we will have an intensity-modulated signal at the output of the PD due to the PM-IM conversion. The recovered microwave signal is given by

$$
\begin{aligned}
V\left(\omega_{\mathrm{e}}, t\right)= & A\left|E_{2}(t)\right|^{2} \\
= & A J_{0}(\gamma) J_{1}(\gamma) \cos \left(\frac{\theta_{1}+\theta_{2}-2 \theta_{0}}{2}-\frac{\pi}{2}\right) \\
& \times \cos \left(\omega_{\mathrm{e}} t+\frac{\theta_{1}-\theta_{2}}{2}\right) \\
= & A J_{0}(\gamma) J_{1}(\gamma) \cos \left(\frac{\chi \lambda^{2} \omega_{\mathrm{e}}^{2}}{4 \pi \mathrm{c}}+\frac{\pi}{2}\right) \\
& \times \cos \left[\left(\omega_{\mathrm{e}} t+L n_{\mathrm{o}} \omega_{\mathrm{e}} / c\right)\right]
\end{aligned}
$$

where $A$ is a constant which is determined by the fiber loss and the responsivity of the $\mathrm{PD}$, and $\chi=D L$ is the dispersion of the fiber loop. Here, the term $L n_{\mathrm{o}} \omega_{\mathrm{e}} / c$ is an additional phase shift introduced and determined by the first time delay; the term $\cos \left(\chi \lambda^{2} \omega_{\mathrm{e}}^{2} / 4 \pi \mathrm{c}+\pi / 2\right)$ is a frequency-dependent $\mathrm{RF}$ loss which is introduced by the third time delay, or the dispersive element [10], [11]. Therefore, due to the PM-IM conversion, an equivalent microwave bandpass filter that is governed by $\cos \left(\chi \lambda^{2} \omega_{\mathrm{e}}^{2} / 4 \pi \mathrm{c}+\pi / 2\right)$ is formed.

The frequency of the $k$ th peak of the response spectrum can be calculated by letting $\chi \lambda^{2} \omega_{\mathrm{e}}^{2} / 4 \pi \mathrm{c}=(2 k-1) \pi / 2, k=$ $1,2,3 \ldots$, that is

$$
\omega_{\mathrm{e}}=\frac{\pi}{\lambda} \sqrt{(2 k-1) \cdot \frac{2 \mathrm{c}}{\chi}} .
$$

The first peak frequency would become the center frequency of the high-Q microwave filter when a two-port PM is used. In fact, the optical field at the output of the PM can be written as

$$
\begin{aligned}
E_{3}(t)=E_{0} \exp \left\{j \left[\omega_{\mathrm{o}} t+V_{\mathrm{e}} \cos \left(\omega_{\mathrm{e}} t\right) / V_{\pi}\right.\right. \\
\left.\left.+V_{\mathrm{e}} \cos \left(\omega_{\mathrm{e}} t+\omega_{\mathrm{e}} \tau\right) / V_{\pi}\right]\right\} \\
=E_{0} \exp \left\{j \left[\omega_{\mathrm{o}} t+2 V_{\mathrm{e}} / V_{\pi}\right.\right. \\
\left.\left.\times \cos \left(\omega_{\mathrm{e}} \tau / 2\right) \cos \left(\omega_{\mathrm{e}} t+\omega_{\mathrm{e}} \tau / 2\right)\right]\right\} \\
=E_{0} \exp \left\{j \left[\omega_{\mathrm{o}} t+\gamma^{\prime}\left(\omega_{\mathrm{e}}\right)\right.\right. \\
\left.\left.\cdot \cos \left(\omega_{\mathrm{e}} t+\omega_{\mathrm{e}} \tau / 2\right)\right]\right\}
\end{aligned}
$$

where $\tau$ is the time delay difference between the two RF ports. By comparing (7) with (1), we may find that the output signal at the output of the two-port PM can be considered as a phase-modulated signal, but with a new phase modulation index given by $\gamma^{\prime}\left(\omega_{\mathrm{e}}\right)=2 V_{\mathrm{e}} \cos \left(\omega_{\mathrm{e}} \tau / 2\right) / V_{\pi}$, which is not a constant but a function of $\omega_{e}$. Therefore, the recovered microwave signal at the output of the PD can also be expressed by (5), but $J_{0}(\gamma) J_{1}(\gamma)$ should be replaced by $J_{0}\left[\gamma^{\prime}\left(\omega_{\mathrm{e}}\right)\right] J_{1}\left[\gamma^{\prime}\left(\omega_{\mathrm{e}}\right)\right]$. Since $J_{0}\left[\gamma^{\prime}\left(\omega_{\mathrm{e}}\right)\right] J_{1}\left[\gamma^{\prime}\left(\omega_{\mathrm{e}}\right)\right]$ can be seen as a quasi-linear function 
when $\gamma^{\prime}\left(\omega_{\mathrm{e}}\right)=2 V_{\mathrm{e}} \cos \left(\omega_{\mathrm{e}} \tau / 2\right) / V_{\pi}$ is less than one, the amplitude of the recovered microwave signal in the case of using a two-port PM will be equal to the amplitude in the case of using a one-port PM times a quasi-sinusoidal periodical function with a period of $2 / \tau$.

Now we can see that the term $J_{0}(\gamma) J_{1}(\gamma)$ in (5) or $J_{0}\left[\gamma^{\prime}\left(\omega_{\mathrm{e}}\right)\right] J_{1}\left[\gamma^{\prime}\left(\omega_{\mathrm{e}}\right)\right]$ is also a frequency-dependent RF loss function which is introduced and determined by the second time delay. As a matter of fact, due to the second time delay, a microwave comb filter with comb spacing of $2 / \tau$ is generated.

Once the loop is closed, the recovered signal at the output of the PD will be amplified and feedback to the PM. The total output at the PD at any instant time is the summation of all circulating fields in the loop, which can be expressed as

$$
\begin{aligned}
V^{\prime}\left(\omega_{\mathrm{e}}, t\right)= & \exp \left(j \omega_{\mathrm{e}} t\right) \times \sum_{m=0}^{\infty}\left\{\mathrm{AG} J_{0}\left[\gamma^{\prime}\left(\omega_{\mathrm{e}}\right)\right] J_{1}\left[\gamma^{\prime}\left(\omega_{\mathrm{e}}\right)\right]\right. \\
& \left.\times \cos \left(\frac{\chi \lambda^{2} \omega_{\mathrm{e}}^{2}}{4 \pi \mathrm{c}}+\frac{\pi}{2}\right)\right\}^{m} \\
& \cdot \exp \left(j m \omega_{\mathrm{e}} \frac{n_{o} L}{\mathrm{c}}\right) \\
= & \exp \left(j \omega_{\mathrm{e}} t\right) \times \sum_{m=0}^{\infty}\left[G_{\mathrm{eff}}\left(\omega_{\mathrm{e}}\right)\right]^{m} \cdot \exp \left(j m \omega_{\mathrm{e}} \frac{n_{o} L}{\mathrm{c}}\right)
\end{aligned}
$$

where $G$ is the gain provided by the RF PA, and $G_{\text {eff }}\left(\omega_{e}\right)$ is the effective open-loop gain, given by

$$
G_{\mathrm{eff}}\left(\omega_{\mathrm{e}}\right)=A G J_{0}\left[\gamma^{\prime}\left(\omega_{\mathrm{e}}\right)\right] J_{1}\left[\gamma^{\prime}\left(\omega_{\mathrm{e}}\right)\right] \cos \left(\frac{\chi \lambda^{2} \omega_{\mathrm{e}}^{2}}{4 \pi \mathrm{c}}+\frac{\pi}{2}\right)
$$

When the effective open-loop gain $G_{\text {eff }}$ is less than unity, (8) can be simplified to

$$
V^{\prime}\left(\omega_{\mathrm{e}}, t\right)=\frac{\exp \left(j \omega_{\mathrm{e}} t\right)}{1-G_{\mathrm{eff}}\left(\omega_{\mathrm{e}}\right) \cdot \exp \left(j \omega_{\mathrm{e}} \frac{n_{\mathrm{o}} L}{\mathrm{c}}\right)} .
$$

The corresponding microwave power $P\left(\omega_{e}, t\right)$ is then given by

$$
P\left(\omega_{\mathrm{e}}, t\right) \propto \frac{1}{1+G_{\mathrm{eff}}^{2}\left(\omega_{\mathrm{e}}\right)-2 G_{\mathrm{eff}}\left(\omega_{\mathrm{e}}\right) \cdot \cos \left(\omega_{\mathrm{e}} \frac{n_{0} L}{\mathrm{c}}\right)} .
$$

Only the frequency component with a phase increment of $L n_{\mathrm{o}} \omega_{\mathrm{e}} / c$ after each loop circulation being a multiple of $2 \pi$ and the total RF loss being minimum in the loop will oscillate. Therefore, when the conditions

$$
\begin{aligned}
& \omega_{\mathrm{e}}=\omega_{\mathrm{osc}}=\frac{4 k_{1}^{\prime} \pi}{\tau}=\frac{\left(2 k_{2}^{\prime}+1\right) \pi c}{n_{\mathrm{o}} L}=\frac{\pi}{\lambda} \sqrt{\frac{2 \mathrm{c}}{\chi}} \\
& \omega_{\mathrm{e}}=\omega_{\mathrm{osc}}=\frac{2\left(2 k_{1}^{\prime \prime}+1\right) \pi}{\tau}=\frac{2 k_{2}^{\prime \prime} \pi c}{n_{\mathrm{o}} L}=\frac{\pi}{\lambda} \sqrt{\frac{2 \mathrm{c}}{\chi}}
\end{aligned}
$$

are satisfied where $k_{1}^{\prime}, k_{2}^{\prime}, k_{1}^{\prime \prime}, k_{2}^{\prime \prime}$ are integers, and the combinations of $k_{1}^{\prime}$ and $k_{2}^{\prime}, k_{1}^{\prime \prime}$ and $k_{2}^{\prime \prime}$ must meet the conditions given in (12a) and (12b), respectively, $\left|G_{\text {eff }}\left(\omega_{e}\right)\right|$ will reach a maximum

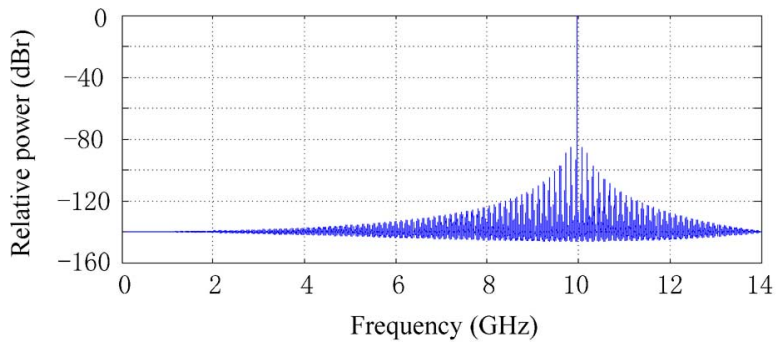

(a)

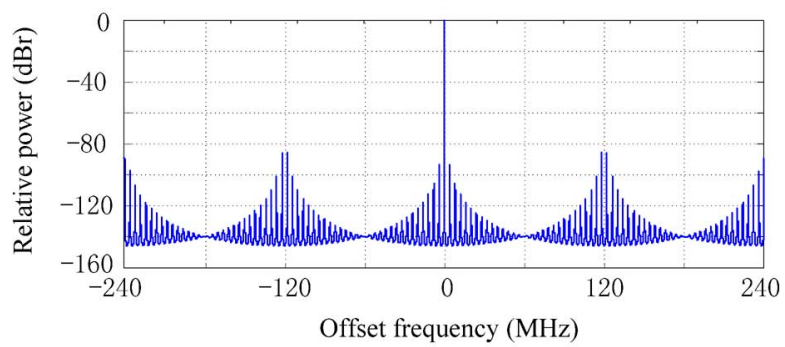

(b)

Fig. 2. (a) Calculated electrical spectrum of the proposed OEO. (b) A close-up view of the calculated electrical spectrum.

and the oscillation mode with a frequency $\omega_{\text {osc }}$ will add up in phase after each circulation. Therefore, $\omega_{\text {osc }}$ would be the oscillation frequency. Substituting (12) into (11) yields

$$
P\left(\omega_{\mathrm{osc}}, t\right) \propto \frac{1}{\left[1-G_{\mathrm{eff}}\left(\omega_{\mathrm{osc}}\right)\right]^{2}} .
$$

Fig. 2 shows the calculated electrical spectrum of the proposed OEO from (11). In the calculation, we chose $G_{\text {eff }}\left(\omega_{\text {osc }}\right)=$ $1-10^{-7}, \chi=623 \mathrm{ps} / \mathrm{nm}, \lambda=1558 \mathrm{~nm}, \tau=8.3 \mathrm{~ns}, n_{o}=$ $1.5, L=50 \mathrm{~m}$, and $V_{e}=0.2 V_{\pi}$ to meet the conditions in (12). The calculated oscillation frequency is about $10 \mathrm{GHz}$.

From Fig. 2, we can see that there is only one strong oscillation mode at about $10 \mathrm{GHz}$ over a frequency span of $14 \mathrm{GHz}$, therefore, single frequency oscillation of the OEO is achieved without using any electrical filters since the whole loop has a frequency response equivalent to a high- $Q$ microwave filter. From Fig. 2(b), the spacing between two adjacent peaks is about $4 \mathrm{MHz}$, which is the mode spacing determined by the first time delay or the overall length of the loop. The spacing between two adjacent envelopes is about $120 \mathrm{MHz}$, which is determined by the second time delay. The highest peak is located at the central frequency of the equivalent microwave bandpass filter realized by the PM-IM conversion in the dispersive element. Thanks to the three different time delays, a frequency response of the OEO with a single frequency having the highest peak over a frequency span of over a few GHz is obtained, which ensures a single-frequency oscillation without the need of a narrowband electronic microwave filter.

From (12), we can see that the oscillation frequency $\omega_{\text {osc }}$ of the OEO is determined by four parameters: the chromatic dispersion $\chi$, the wavelength of the incident light wave $\lambda$, the time delay $\tau$ and the loop length $L$. Under the conditions given in 
(12), $\omega_{\text {osc }}$ can be continuously tunable by changing $\chi, \lambda, \tau$ or $L$. In particular, if $\tau$ and $L$ are set and satisfy

$$
\frac{1}{\tau}=p \cdot \frac{c}{2 n_{\mathrm{O}} L}, p=1,2,3 \ldots
$$

(in practice, $L$ is very large so that we have $1 / \tau \gg c / \mathrm{nL}$ ), based on (12) when $k_{1}^{\prime}$ and $k_{1}^{\prime \prime}$ are increased by one, we can have

$$
\begin{aligned}
\omega_{\mathrm{osc}}^{\prime} & =\frac{4\left(k_{1}^{\prime}+1\right) \pi}{\tau}=\frac{\left[2\left(k_{2}^{\prime}+p\right)+1\right] \pi c}{n_{\mathrm{o}} L}=\frac{\pi}{\lambda^{\prime}} \sqrt{\frac{2 \mathrm{c}}{\chi^{\prime}}} \\
& =\omega_{\mathrm{osc}}+2 \pi \cdot \frac{2}{\tau}
\end{aligned}
$$

or

$$
\begin{aligned}
\omega_{\mathrm{osc}}^{\prime} & =\frac{2\left[2\left(k_{1}^{\prime \prime}+1\right)+1\right] \pi}{\tau}=\frac{2\left(k_{2}^{\prime \prime}+p\right) \pi c}{n_{\mathrm{o}} L}=\frac{\pi}{\lambda^{\prime}} \sqrt{\frac{2 \mathrm{c}}{\chi^{\prime}}} \\
& =\omega_{\mathrm{osc}}+2 \pi \cdot \frac{2}{\tau}
\end{aligned}
$$

where $\omega_{\text {osc }}^{\prime}$ is the new oscillation angular frequency when $\lambda$ and $\chi$ are tuned to $\lambda$, and $\chi^{\prime}$, and $\omega_{\mathrm{Qsc}}^{\prime}$ is increased by $2 \pi \cdot(2) /(\tau)$. Considering that when $k_{1}^{\prime}=k_{1}$, we have

$$
\frac{4 k_{1}^{\prime} \pi}{\tau}+2 \pi \cdot \frac{1}{\tau}=\frac{2\left(2 k_{1}^{\prime \prime}+1\right) \pi}{\tau}
$$

we come to a conclusion that the oscillation frequency will be tuned at a step of $1 / \tau$ by only changing the value of $\chi$ or $\lambda$, when the conditions in (12) are satisfied, and $\tau$ and $L$ are set to meet the condition given in (14). Based on (12), we have

$$
\Delta \omega_{\mathrm{osc}}=-\frac{\pi}{\lambda} \sqrt{\frac{2 \mathrm{c}}{\chi}} \cdot \frac{\Delta \lambda}{\lambda}-\frac{\pi}{2 \lambda} \sqrt{\frac{2 \mathrm{c}}{\chi}} \cdot \frac{\Delta \chi}{\chi} .
$$

In practice, the relative variation of the wavelength $\Delta \lambda / \lambda$ is much smaller than that of the dispersion $\Delta \chi / \chi$. Therefore, the dispersion tuning can be employed for coarse frequency tuning and the wavelength tuning can be employed for fine tuning. The dependence of the oscillation frequency on the dispersion and the wavelength is calculated and shown in Fig. 3. From Fig. 3, we can clearly see that the oscillation frequency will decrease when the value of $\lambda$ or $\chi$ is increased. The wavelength tuning leads to smaller oscillation frequency change than the dispersion tuning, due to a larger relative variation of the dispersion. The use of an LCFBG with a dispersion $\chi$ from a few hundreds to a few thousands $\mathrm{ps} / \mathrm{nm}$, a frequency tunable range that is more than $10 \mathrm{GHz}$ can be achieved.

\section{EXPERIMENTS}

Experiments based on the setup shown in Fig. 1 are performed. Since a two-port PM is not available, in the experiments the two-port PM is realized by cascading two one-port PMs. The dispersive element used in the experiments is a LCFBG with a chromatic dispersion $\chi$ at $1558 \mathrm{~nm}$ is about $623 \mathrm{ps} / \mathrm{nm}$. The power of the incident light wave to the PM is about $10 \mathrm{dBm}$. The power of the light wave sent to the PD is about $3 \mathrm{dBm}$. The total length of the OEO loop is approximately 50 meter and $\tau$ is about

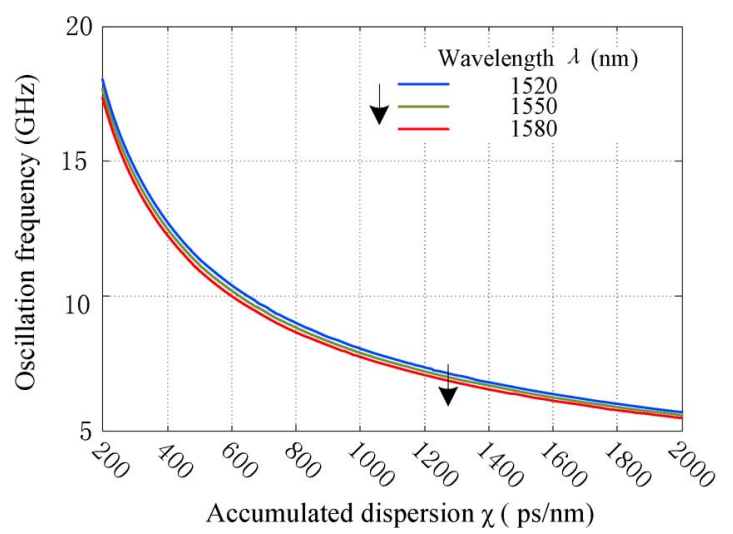

Fig. 3. Dependence of the oscillation frequency on the wavelength and the dispersion.

$8.3 \mathrm{~ns}$. The wavelength of the light wave is set at $1558 \mathrm{~nm}$. With the parameters given, the calculated oscillation frequency would be approximately equal to $10 \mathrm{GHz}$. When the loop is closed, the OEO starts to oscillate and a single-frequency oscillation is observed. The experimental results are shown in Fig. 4.

Fig. 4(a) shows the spectrum of the generated microwave signal at $10 \mathrm{GHz}$ with a frequency span of $20 \mathrm{GHz}$. As can be seen the OEO is operating at single-frequency oscillation mode with a oscillating frequency of $10 \mathrm{GHz}$, which agrees well with the theoretical value. To have a better view of the spectrum, a smaller frequency span of $200 \mathrm{kHz}$ is used and the spectrum is shown in Fig. 4(b). Fig. 4(c) shows the optical spectrum at the output of the PM. As expected, stable and clear sidebands are observed due to the single-frequency oscillation.

To evaluate the phase noise performance, we measure the single-sideband phase noise of the generated $10 \mathrm{GHz}$ signal, which is shown in Fig. 4(d). The theoretical phase noise when the total loop length is about 50 meters [1] is also shown. As can be seen the generated signal has a phase noise of about -100 $\mathrm{dBc} / \mathrm{Hz}$ at an offset of $10 \mathrm{kHz}$. When the offset frequency is lower than $1 \mathrm{kHz}$, the phase noise performance is much poorer, which may be caused due to the following reasons [1], [12]: 1) the polarization drifts of the light wave launched into the PM. Since the PM is a polarization-dependent device, the polarization drifts of the light wave will cause the power drifts of the light wave in the loop, leading to the power fluctuations of the generated signal; 2) the fiber loop vibrations and the environmental temperature change; 3 ) the variations of the RF delay $\tau ; 4)$ the current fluctuations of the pump current applied to the EDFA. The pump current variations will lead to the change of the loop gain, which would lead to the power fluctuations and increase phase noise of the generated microwave signal.

The peaks observed at the frequencies beyond $1 \mathrm{MHz}$ are the other oscillation modes. The peak spacing is equal to the mode spacing which is about $4 \mathrm{MHz}$ determined by the total length of the loop or the first time delay.

The phase noise of the generated signal in our system is not as good as that reported in [1], [6], [9]. The fundamental reason of the poorer phase noise performance is due to the short total loop length, which is about 50 meters. In [1], [6], [9], the loop 


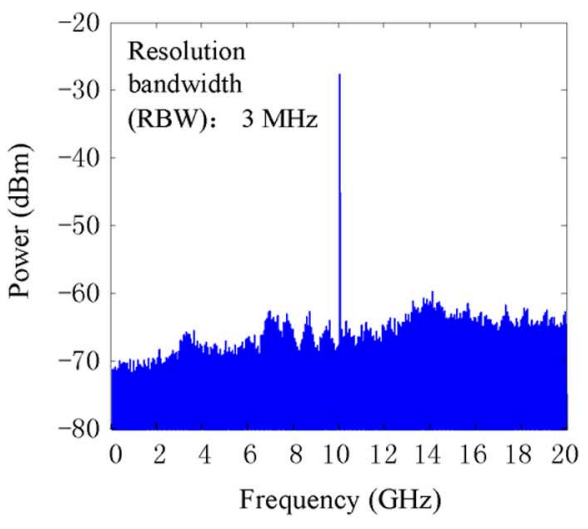

(a)

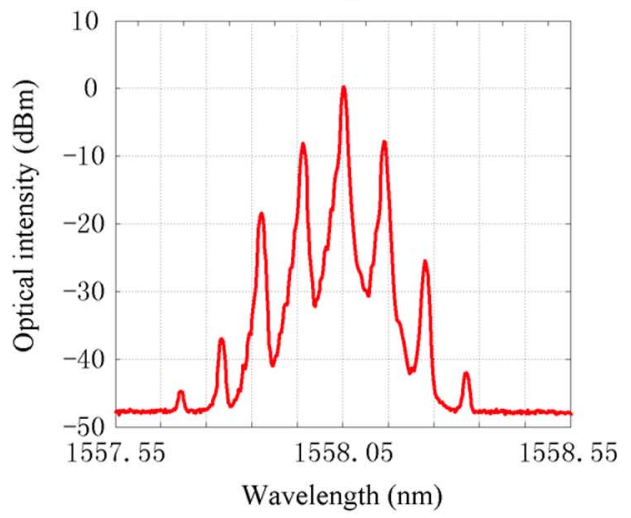

(c)

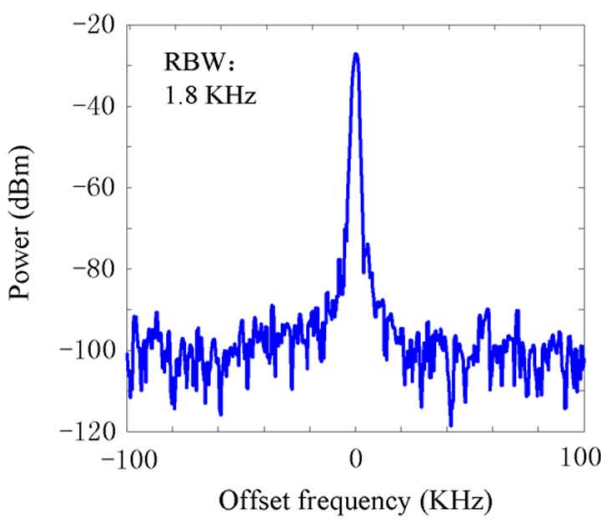

(b)

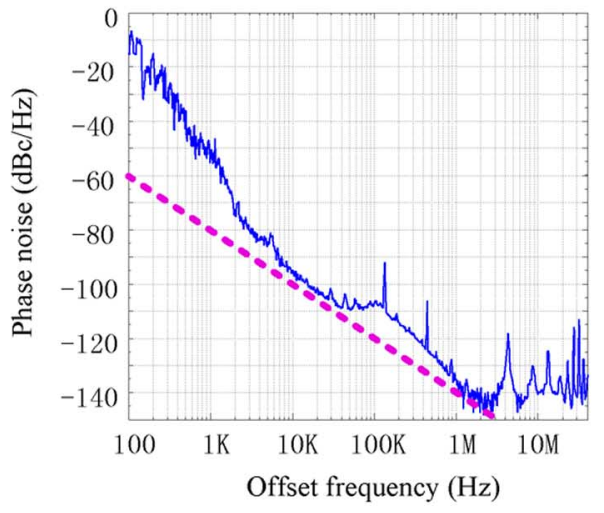

(d)

Fig. 4. Experimental results showing the single-frequency oscillation of the proposed OEO. Measured electrical spectrum of the generated 10-GHz signal with a frequency span of (a) $20 \mathrm{GHz}$, (b) $200 \mathrm{kHz}$. (c) Measured optical spectrum of the modulated light wave at the output of the PM. The RBW is $0.01 \mathrm{~nm}$. (d) Measured phase noise of the generated $10 \mathrm{GHz}$ signal (blue line) and the theoretical phase noise (purple-dotted line).

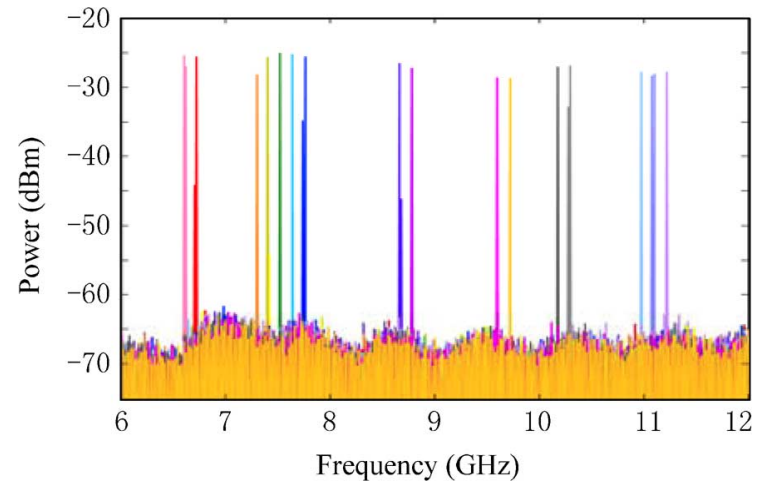

Fig. 5. Measured electrical spectra of the generated microwave signal at different oscillation frequency. The minimum line spacing is constant, and equal approximately to $120 \mathrm{MHz}$, which is the tuning step.

length is at least a few kilometers. The phase noise performance can be improved by employing a loop with a longer length.

The stability of the generated signal in term of mode hopping is also investigated. To do so, we allow the system to operate in a room temperature for a few hours, the oscillation frequency is kept constant, with no mode hopping observed.

The frequency tunability of the OEO is also investigated. We demonstrate the frequency tunability in a particular case where condition (14) is met. The frequency of the generated signal is tuned at a step of $1 / \tau$, or approximately $120 \mathrm{MHz}$, by changing the value of $D$ or $\lambda$. Fig. 5 shows the spectrum of the generated microwave signal with its frequency tuned over a frequency range from about 6.5 to $11.5 \mathrm{GHz}$. The tuning step is $120 \mathrm{MHz}$. The frequency tuning range can be further increased by increasing the tunable ranges of $\chi$ and $\lambda$.

\section{CONCLUSION}

A novel approach to achieving a frequency-tunable OEO using a two-port PM and a dispersive element was proposed and experimentally demonstrated. Due to the PM-IM conversion in the dispersive element, the entire OEO loop had a frequency response corresponding to a high-Q microwave filter to support only single-frequency oscillation. The frequency of the generated microwave signal could be easily tuned by tuning the wavelength of the laser source or the dispersion of the dispersive element. A frequency tunable range as large as a few $\mathrm{GHz}$ was achieved. The generated microwave signal exhibited a good phase noise performance with a phase noise of $-100 \mathrm{dBc} / \mathrm{Hz}$ at an offset of $10 \mathrm{kHz}$. The phase noise performance can be further improved if a longer loop is employed. The key significance of the proposed technique is that no electronic microwave filters are needed which ensures a large frequency tuanble range through all-optical tuning. The oscillation frequency of the OEO could also be continuously tuned by changing the time delay difference introduced by the DL or the length of the OEO loop. 


\section{REFERENCES}

[1] X. S. Yao and L. Maleki, "Optoelectronic microwave oscillator," J. Opt. Soc. Amer. B, vol. 13, no. 8, pp. 1725-1735, Aug. 1996.

[2] L. Huo, Y. Dong, C. Y. Lou, and Y. Z. Gao, "Clock extraction using an optoelectronic oscillator from high-speed NRZ signal and NRZ-to-RZ format transformation," IEEE Photon. Technol. Lett., vol. 15, no. 7, pp. 981-983, Jul. 2003.

[3] H. Tsuchida and M. Suzuki, "40-Gb/s clock recovery using an injection-locked optoelectronic oscillator," IEEE Photon. Technol. Lett., vol. 17, no. 1, pp. 211-213, Jan. 2005.

[4] X. S. Yao and L. Maleki, "Opto-electronic oscillator and its applications," in Proc. MWP, 1996, pp. 265-268.

[5] D. Eliyahu and L. Maleki, "Tunable, ultra-low phase noise YIG based opto-electronic oscillator," in Proc. IEEE MTT-S Int. Microwave Symp. Dig., 2003, pp. 2185-2187.

[6] X. S. Yao and L. Maleki, "Multiloop optoelectronic oscillator," IEEE J. Quantum Electron., vol. 36, no. 1, pp. 79-84, Jan. 2000.

[7] Y. Jiang, J. Yu, Y. Wang, L. Zhang, and E. Yang, "An optical domain combined dual-loop optoelectronic oscillator," IEEE Photon. Technol. Lett., vol. 19, no. 11, pp. 807-809, Jun. 2007.

[8] E. Shumakher and G. Eisenstein, "A novel multiloop optoelectronic oscillator," IEEE Photon. Technol. Lett., vol. 20, no. 22, pp. 1881-1883, Nov. 2008.

[9] S. Poinsot;, H. Porte, J. Goedgebuer, W. T. Rhodes, and B. Boussert, "Continuous radio-frequency tuning of an optoelectronic oscillator with dispersive feedback," Opt. Lett., vol. 27, no. 15, pp. 1300-1302, Aug. 2002.

[10] F. Zeng and J. P. Yao, "All-optical bandpass microwave filter based on an electro-optic phase modulator," Opt. Exp., vol. 12, no. 16, pp. 3814-3819, Aug. 2004.

[11] F. Zeng and J. P. Yao, "Investigation of phase modulator based alloptical bandpass microwave filter," J. Lightw. Technol., vol. 23, no. 4, pp. 1721-1728, Apr. 2005.

[12] D. Eliyahu, D. Seidel, and L. Maleki, "RF amplitude and phase-noise reduction of an optical link and an opto-electronic oscillator," IEEE Trans. Microw. Theory Tech., vol. 56, no. 2, pp. 449-456, Feb. 2008.
Wangzhe Li (S'08) received the B.Eng. degree in electronic science and technology from Xi' an Jiaotong University, Xi'an, China, in 2004, and the M.Sc. degree in optoelectronics and electronic science from Tsinghua University, Beijing, China, in 2007. He is currently working toward the Ph.D. degree in electrical engineering in the Microwave Photonics Research Laboratory, School of Information Technology and Engineering, University of Ottawa, Ottawa, ON, Canada.

His current research interests include photonic generation of microwave and $\mathrm{THz}$ signals, semiconductor lasers and laser arrays, and photonics ADC.

Jianping Yao (M'99-SM'01) received the Ph.D. degree in electrical engineering from the Université de Toulon, Toulon, France, in 1997.

He joined the School of Information Technology and Engineering, University of Ottawa, Ottawa, ON, Canada, in 2001, where he is currently a Professor, Director of the Microwave Photonics Research Laboratory, and Director of the Ottawa-Carleton Institute for Electrical and Computer Engineering. From 1999 to 2001, he held a faculty position with the School of Electrical and Electronic Engineering, Nanyang Technological University, Singapore. He holds a Yongqian Endowed Visiting Chair Professorship with Zhejiang University, China. He spent three months as an Invited Professor with the Institut National Polytechnique de Grenoble, France, in 2005. He has authored or coauthored over 280 papers including 160 papers in peer-reviewed journals and 120 papers in conference proceeding. His research has focused on microwave photonics, which includes all-optical microwave signal processing, photonic generation of microwave, mm-wave and $\mathrm{THz}$, radio over fiber, UWB over fiber, fiber Bragg gratings for microwave photonics applications, and optically controlled phased array antenna. His research interests also include fiber lasers, fiber-optic sensors and bio-photonics. He is an associate editor of the International Journal of Microwave and Optical Technology.

Dr. Yao is a Fellow of the Optical Society of America and a senior member of IEEE Photonics Society and IEEE Microwave Theory and Techniques Society. He is a Registered Professional Engineer of Ontario. He is on the editorial board of the IEEE TRANSACTIONS ON MiCROWAVE THEORY AND TECHNIQUES. He was the recipient of the 2005 International Creative Research Award of the University of Ottawa, the 2007 George S. Glinski Award for Excellence in Research, and an NSERC Discovery Accelerator Supplements Award in 2008. He was named University Research Chair in Microwave Photonics in 2007. 